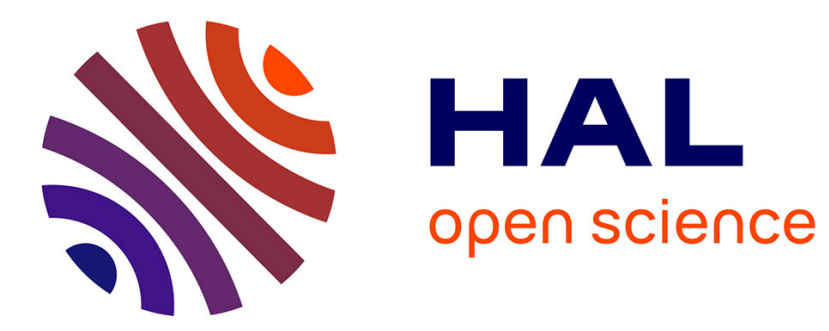

\title{
Maintenance decision making tool reaching a compromise between maintainability and reliability performances
}

Edouard Thomas, Eric Levrat, Benoît Iung

\section{- To cite this version:}

Edouard Thomas, Eric Levrat, Benoît Iung. Maintenance decision making tool reaching a compromise between maintainability and reliability performances. 17th IFAC World Congress, Jul 2008, Séoul, South Korea. pp.907-912, 10.3182/20080706-5-KR-1001.2477 . hal-00202686

\section{HAL Id: hal-00202686 \\ https://hal.science/hal-00202686}

Submitted on 7 Jan 2008

HAL is a multi-disciplinary open access archive for the deposit and dissemination of scientific research documents, whether they are published or not. The documents may come from teaching and research institutions in France or abroad, or from public or private research centers.
L'archive ouverte pluridisciplinaire HAL, est destinée au dépôt et à la diffusion de documents scientifiques de niveau recherche, publiés ou non, émanant des établissements d'enseignement et de recherche français ou étrangers, des laboratoires publics ou privés. 


\title{
Maintenance decision making tool reaching a compromise between maintainability and reliability performances
}

\author{
Edouard Thomas, Eric Levrat, Benoît Iung \\ Research Centre for Automatic Control (CRAN) \\ Nancy University, UMR 7039 CNRS UHP INPL \\ Faculty of Sciences BP 239 \\ 54506 Vandoeuvre cedex (France) \\ (emails: \{edouard.thomas, eric.levrat, benoit.iung\}@cran.uhp-nancy.fr)
}

\begin{abstract}
An original maintenance decision making tool based upon Bruss theorem has previously been investigated by the authors to select the optimal last production stoppage convenient to operate a maintenance action on a component according to its degradation conditions. This stoppage is optimal with respect to antagonistic criteria such as maintainability and reliability combined. The approach is also opportunistic in the sense that maintenance is developed during production stoppages already planned. However the optimality with respect to the separate criteria alone has not been taken into account to find this global optimal stoppage. The present work aims at providing, for this tool, a way to eliminate stoppages a priori unacceptable for one criterion, so that this stoppage cannot be proposed as the final global solution. This will be done with the help of a maintenance expert. Case studies are presented and commented. Two criteria have been considered, namely maintainability and reliability, which are two key elements to bring together to perform a maintenance action. These criteria will also be used for emphasis on the difference between local optimal decisions and a global optimal decision.
\end{abstract}

\section{INTRODUCTION}

In a lifecycle maintenance concept, the characteristics of the manufactured product have a huge influence on the performances of the manufacturing system (Takata, et al., 2004). From a global perspective of lifecycle management, the new role of the maintenance process is now to preserve the characteristics of the product (Cunha and CaldeiraDuarte, 2004). This concept is part of a culture which defends that maintenance activities should be of equal importance to actual production activities, since maintenance has to be considered not only in the 'Production' phase, but also all along the product life cycle (Van Houten, et al., 1998). Thus to enable the enhancement of the maintenance role, the integration of the maintenance planning into the production planning has to be considered (Levrat, et al., 2007a). However a unified maintenance-production framework does not yet exist. The goal is to develop opportunistic maintenance actions synchronised with production so as to guarantee the performances of both the equipment and of the manufactured product. From the point of view of the maintenance strategy, it leads to move from conventional maintenance strategies (such as corrective maintenance or scheduled preventive maintenance) to condition-based or predictive maintenance strategies. These are performed on a component only when a certain level of degradation (which would impact the conditions of the product) is reached. To introduce such strategies, the maintenance expert needs to have at his disposal just-in-time information provided by a prognosis process (Iung, et al., 2005) in order to assess new situations for the product and the system, and to anticipate the possible deteriorations of the product as well as the failures of the system. More precisely, in relation to (ISO13381-1, 2004), the prognosis process should be used in order to foresee how the characteristics of a component will evolve until its failure, then until the breakdown of the system. This provides a remaining useful life (RUL) for this component, and therefore a temporal horizon to perform some predictive maintenance actions on this component. Thus a new maintenance issue is to consider this just-in-time information to propose opportunistic preventive maintenance actions, in the sense that planned production stoppages within this temporal horizon could be used to develop these actions. It can be summarised as follows: taking into account the RUL of a component, is it possible to select one of the forecast production stoppages in order to carry out a maintenance action that would reach a compromise between safety, performances of the product, spare parts availability, reliability and maintainability of the component...? If so, how to classify the selected production stoppages in decreasing order of relevance according to these criteria? Such a sorting would use dynamic information related to components and to production stoppages. In relation to this issue, some contributions have already been achieved. They propose either (a) common maintenance-production scheduling (Kianfar, 2005), but which does not consider the case where production stoppages are imposed; or (b) selection of a production stoppage based on expert judgement (Rosqvist, 2002), but which is subjective and can be nonoptimal; or finally (c) the decreasing of the maintenance operation time or cost-oriented approaches (Gharbi and Kenné, 2005), but which does not take into consideration just-in-time information related to the component while 
integrating some items not solved by the previous approaches. The originality of the approach presented in (Levrat, et al., 2007a) and based upon Bruss algorithm is first to keep the initial production scheduling without modifying it, second to ensure the optimality of the maintenance decision with respect to a criterion, and third to use current system information delivered by a prognosis process. Indeed Bruss algorithm selects, for a component, the last optimal production stoppage already planned that is relevant to perform a predictive maintenance action on this component. For this maintenance issue, the optimality of Bruss algorithm holds with respect to the combination of antagonistic criteria inside one unique function. It thus provides a first global (macroscopic) idea of the solution sought by the expert. However, such a decision can be unacceptable for him. For instance, consider a stoppage for which the reliability of the component will be 'very high' and its maintainability 'very low': such a stoppage could be selected by the algorithm although it is 'too short' to perform the maintenance action (see stoppage 15 in sections 3 and 4). Therefore the use of Bruss algorithm alone unveils the following problem: solutions that are unacceptable according to one criterion can be acceptable as a global decision. This is the issue that is dealt with in the present work. The elimination of such unacceptable stoppages involves the expert who will specify a level of demands for each criterion: any stoppage whose probability of success is lower than this level (or threshold) will be eliminated. This threshold enables the expert to integrate some of his knowledge into the model. Moreover the threshold is directly linked to the risk the expert can accept for a stoppage to be a success with respect to the considered criterion. In the present paper, two relevance criteria related to the maintenance action will be considered: maintainability and reliability (any other two antagonistic criteria could be used). The main results are first that optimal decisions, with respect to one criterion, can be irrelevant for the global maintenance purpose, next that non-optimal local decisions can be appreciated global solutions for such a multi-criteria approach. The differences between optimal maintenance decisions (with respect to each criterion) and a global optimal decision (taking into account both criteria) will then be emphasised: through a level of requirements for each criterion, the expert will be able to eliminate those stoppages that are a priori unacceptable for this criterion. This phase is inherent to the industrial and scientific problem that is considered, and not to the approach, method or tool adopted. It represents a first step towards the formalisation of the expert's own knowledge and experience related to the components, the stoppages, the environment... The rest of the paper is organised as follows: section 2 briefly presents Thomas Bruss' results and how his algorithm can be used. Section 3 presents optimality of the decision with respect to each criterion, and section 4 proposes a global optimum involving both criteria.

\section{BRUSS ALGORITHM}

Thomas Bruss' theorem (Bruss, 2000) is briefly presented in this section. It is based upon the theory of optimal stopping (Chow, et al., 1991). The 'odds algorithm' is a mathematical decision making tool derived from these results. This tool indicates the optimal behaviour in some situations where future is uncertain. Some applications are presented.

\subsection{Statement of the problem}

A component of a production system being given, the prognosis process provides for this component, at instant 0 , a RUL of $T$ time units, thus defining a bounded observation horizon $[0 ; T]$. The expert has at his disposal, before instant $T$, the list of the $n$ planned production stoppages. These are scheduled by the production department and defined by the means of 'beginning instants' (an increasing sequence $\left(a_{i}\right)_{1 \leq i \leq n}$, where $\left.a_{i} \in[0 ; T], 1 \leq i \leq n\right)$ and of respective 'durations' $d_{i}>0,1 \leq i \leq n$. Among these production stoppages, some will be appropriate to develop predictive maintenance actions. In the following sections, a stoppage will be appropriate if its duration is 'long enough' (maintainability point of view), or if its beginning instant comes 'soon enough' (reliability point of view), or if its duration is 'long enough' and its beginning instant comes 'soon enough' (maintainability and reliability point of view). These privileged stoppages will be called 'successes' in the sequel. Thus the main issue can be formulated this way: determine the last success at which a predictive maintenance action can be performed in order to restore a given component into a nominal state, for preserving the expected product conditions.

\subsection{Thomas Bruss'general results}

Bruss theorem answers the question of finding a priori (at instant 0) the last success, among a finite number of events to happen, in a given bounded horizon. 'Successes', in this general formulation, are defined thanks to conditions on the events (for example 'Getting a six when rolling a fair die', or 'Performing a maintenance action when the stoppage comes'). Bruss' result can be formulated as follows: let $A_{1} \ldots A_{n}$ be $n$ independent random variables defined on the same probability space ( $n$ is the number of die throws, or the number of planned production stoppages). These variables are observed sequentially (i.e. one by one, from $A_{1}$ to $A_{n}$ ). It is assumed that it is possible to stop the observation on any variable of this sequence, without recalling on the previous ones. A 'success' will be any specific realisation of an observation, as stated above. The goal is to find a stopping rule that will maximise the probability to stop the observation precisely on the last success of the sequence (for example, stop the observation precisely at the appearance of the last ' 6 ' of the sequence). It will be with respect to the maximisation of this probability that a production stoppage or a strategy will be characterised as 'optimal' in the sequel. The maximisation of this probability allows the frame of an optimal strategy. The following quantities will be used:

$$
p_{i}:=P\left(A_{i}\right), \quad q_{i}:=1-p_{i}, \quad r_{i}:=p_{i} / q_{i}, \quad 1 \leq i \leq n .
$$

The quantities $r$ are traditionally called the 'odds'. The theorem can now be stated: an optimal rule for finding the 
last success exists, and consists in stopping on the first index $k$ (if any) with $A_{k}$ a success and $s \leq k \leq n$, where

$$
s:=\sup \left(1 ; \sup \left\{1 \leq j \leq n \mid \sum_{i=j}^{n} r_{i} \geq 1\right\}\right)
$$

with the convention $\sup \{\varnothing\}=-\infty$. The optimal reward associated to this rule (i.e. the win probability associated to this strategy) is $\left(\prod_{i=s}^{n} q_{i}\right) \cdot\left(\sum_{i=s}^{n} r_{i}\right)$. Moreover it is proved in (Bruss, 2003) that this optimal reward is greater than $36.75 \%$ if $\sum_{i=s}^{n} r_{i} \geq 1$.

For the maintenance problem considered in the present paper, the random variable $A_{i}(1 \leq i \leq n)$ represents the occurrence of a success for the $i^{\text {th }}$ production stoppage to support the development of a maintenance action. Its probability $p_{i}$ will be calculated thanks to a distribution $X$ which will successively represent the maintainability of the component during the stoppage $A_{i}$ (subsection 3.2), the reliability of the component at the beginning of the stoppage $A_{i}$ (subsection $3.3)$, and the product of the two previous ones (section 4). Its 'odd' will thus be equal to $r_{i}=X\left(A_{i}\right) /\left(1-X\left(A_{i}\right)\right)$.

\subsection{Applications}

An algorithm adapted from (Bruss, 2000) has been conceived to use this result for the particular issue tackled in this paper. The following assumption is convenient: the variables $A_{1} \ldots A_{n}$ are independent random variables. This assumption can be justified that way: the stoppages of the system are subject to many constraints, ranging from production (requirements for the quantity of products to be transformed by the system), to management (requirements for the use of the system) and legal recommendations (to respect safety standards). With this assumption, the 'odds algorithm' has been used and generalised in many ways: in (Thomas, et al., 2006), the algorithm has been used to find the optimal production stoppage and the associated win probability, in the case where a success is defined thanks to reliability and maintainability combined. The reliability function was Weibull-like, and the maintainability function was an exponential distribution. The influence of the parameters of these distributions on the optimal stoppage was thoroughly studied. In (Thomas, et al., 2007), the algorithm was used recursively to classify all the production stoppages in decreasing order of relevance, for two components of the same system with the same RUL. This study allowed to consider an opportunistic maintenance intervention, by carrying out the two maintenance actions during one appropriate production stoppage. In (Iung, et al., 2007) and (Levrat, et al., 2007b), the algorithm was used on an industrial case, to classify the production stoppages by decreasing order of relevance, according to reliability and performances. Those studies and results were all recorded in
(Levrat, et al., 2007a). They provide in every case an optimal solution combining different antagonistic criteria. In the following sections, the way to consider optimality with respect to each criterion, in order to take the final global decision, will be discussed.

\section{OPTIMALITY WITH RESPECT TO MAINTAINABILITY OR RELIABILITY}

Bruss algorithm will be recursively applied on academic examples in this section, where successes will be defined in terms of maintainability and reliability respectively.

\subsection{Technical data and directions for use}

The following characteristics will be used in this section: the RUL of the component considered is $T=800 \mathrm{~h}$, the observation horizon is thus $[0 \mathrm{~h} ; 800 \mathrm{~h}]$; the number of production stoppages planned in the horizon is $n=20$; the maintainability of this component is supposed to follow an exponential distribution with parameter $0.2 \mathrm{~h}^{-1}$ (the mean time to repair is thus equal to $5 \mathrm{~h}$ ); its reliability is supposed to be Weibull-like, with 1.5 for the shape parameter, $600 \mathrm{~h}$ for the scale parameter and $0 \mathrm{~h}$ for the location parameter. The beginnings and the durations of the stoppages are indicated in hours (h); ' $0.5 \mathrm{~h}$ ' means for example 30 minutes.

In the following tables (Tables 1, 2 and 3), the production stoppages are listed by decreasing order of relevance with respect to the aforementioned characteristics (the stoppages are the same, only the criterion is different). Successes are defined by the expert who provides a threshold (in $[0 ; 1]$ ) corresponding to his own demands, according to his experience, his knowledge or his know-how. The higher the threshold, the more demanding the expert. The lower the threshold, the less exigent the expert. A threshold that is too high will lead to select no stoppages, as none of them will fulfill the demands of the expert. This threshold corresponds to the risk that the expert does tolerate concerning the maintenance action. Indeed only he can decide whether the risk not to be able to perform the maintenance action at the due date (reliability criterion) or/and during the expected duration (maintainability criterion) is acceptable or not. The meaning of the threshold is not the same for two different criteria and sums up the expert's knowledge concerning the criticality of the component, the expected performances of the product to be manufactured, the working environment, the safety... Whatever the threshold, the algorithm shall propose decisions (optimal or degraded with warnings). Indeed the algorithm is first used to find the last success, convenient (in accordance with the threshold) for the maintenance action. This decision is optimal with respect to the criterion considered. But if the corresponding proposed stoppage is to be abandoned by the expert, for any reason he would have, then Bruss algorithm is used again, as in (Thomas, et al., 2007), to select, among the remaining stoppages, the optimal decision (with respect to the same criterion, with the same threshold). Therefore the algorithm can be used recursively to classify all the production stoppages by decreasing order of relevance. Three different 
kinds of decision are actually proposed by the algorithm. They are, by decreasing order of optimality, and independently for each of the lines of the following tables ( $m$ a positive integer lower or equal than $n$ ):

1. 'Optimal choice number $m$ ': optimal decision provided by the 'odds algorithm'. In the table, all the corresponding columns are filled in. The demands from the expert are satisfied. Such a stoppage should be considered.

2. 'Choice number $m$ ': optimal stoppage for Bruss algorithm, but the threshold demanded by the expert is too high. The optimal stoppage is proposed, but in the table no probabilities are filled in (symbol *). Such a stoppage can be selected only if the threshold is not of great importance or relevance. A nonoptimal preceding stoppage will yet fulfill the requirements of the expert.

3. 'Degraded choice number $m$ ': the total sum of all the odds has not reached the value 1 , or the threshold demanded by the expert is too high for a stoppage that is not optimal (for Bruss algorithm). However the algorithm proposes a degraded decision. These degraded decisions are marked with a symbol / . The expert should propose a lower threshold, or modify the characteristics of the stoppages.

\subsection{Results concerning the maintainability alone}

The beginnings of the production stoppages do not interfere in the context of the maintainability alone. Therefore these could occur at any time. For the sakes of simplicity and consistency with the next section, the dates of beginnings are supposed to be regularly distributed on the horizon, from 30 hours to 30 hours. For instance, stoppage 1 will begin at instant $t=30 \mathrm{~h}$, stoppage 2 at $t=60 \mathrm{~h}$ and stoppage 20 at $t=600 \mathrm{~h}$. In Table 1, the stoppages are listed by decreasing order of relevance according to maintainability. This list is obtained by applying recursively Bruss algorithm (inferred from the results of subsection 2.2) to these stoppages. The purpose is to choose the last stoppage that will be a success, in terms of maintainability, with the threshold imposed by the expert. More precisely, the first column lists the ranking of the alternatives (by decreasing order of relevance). The second column presents the number of the stoppages associated to these ranks. The third column provides the probabilities associated to the strategy proposed by Bruss theorem for these ranks. The fourth column provides the probabilities that the corresponding stoppages be successes (assessed by using function $X$; see subsection 2.2). The fifth column (which does not exist in Table 3) specifies the durations of the corresponding stoppages in Table 1 and the beginnings of the corresponding stoppages in Table 2. Indeed the maintainability is related to the durations of the stoppages (present subsection 3.2) and the reliability is related to their instants of beginning (subsection 3.3). As explained in subsection 3.1, two lines in a same table (Tables 1 to 3 ) are independent (they relate to different problems, as the stoppages are not the same). In particular, it generally does not make sense to compare their respective probabilities.

\begin{tabular}{|c|c|c|c|c|}
\hline Rank & $\begin{array}{c}\text { Proposed } \\
\text { stoppage }\end{array}$ & $\begin{array}{c}\text { Strategy } \\
\text { probability }\end{array}$ & $\begin{array}{c}\text { Stoppage } \\
\text { probability }\end{array}$ & $\begin{array}{c}\text { Stoppage } \\
\text { duration } \\
\text { (hours) }\end{array}$ \\
\hline 1 & 20 & 0.51 & 0.51 & 3.6 \\
\hline 2 & 19 & 0.54 & 0.54 & 3.9 \\
\hline 3 & 18 & 0.54 & 0.54 & 3.9 \\
\hline 4 & 16 & 0.50 & 0.52 & 3.7 \\
\hline 5 & 17 & $*$ & $*$ & 3.4 \\
\hline 6 & 14 & 0.50 & 0.50 & 3.5 \\
\hline 7 & 13 & $*$ & $*$ & 3.3 \\
\hline 8 & 12 & 0.50 & 0.52 & 3.7 \\
\hline 9 & 11 & $*$ & $*$ & 3.2 \\
\hline 10 & 10 & $*$ & $*$ & 3.2 \\
\hline 11 & 9 & $*$ & $*$ & 3.3 \\
\hline 12 & 8 & $*$ & $*$ & 3.4 \\
\hline 13 & 7 & 0.50 & 0.52 & 3.7 \\
\hline 14 & 6 & 0.50 & 0.50 & 3.5 \\
\hline 15 & 5 & 0.50 & 0.54 & 3.8 \\
\hline 16 & 4 & $*$ & $*$ & 3.4 \\
\hline 17 & 3 & 0.50 & 0.52 & 3.7 \\
\hline 18 & 15 & $*$ & $*$ & 3.2 \\
\hline 19 & 1 & 0.50 & 0.54 & 3.8 \\
\hline 20 & 2 & $/$ & $/$ & 2.0 \\
\hline
\end{tabular}

Table 1. Proposed stoppages for the maintainability alone

In Table 1, the threshold has been chosen equal to 0.50 by the expert. The mean time to repair is equal to $5 \mathrm{~h}$, and the durations belong to [3h; $4 \mathrm{~h}]$. Such a table should be interpreted that way: the optimal last stoppage relevant to perform the maintenance action is the twentieth. Selecting this stoppage is the best strategy, and the win probability associated to this strategy is 0.51 (assessed thanks to Bruss theorem). The probability that the stoppage allow to perform the maintenance action is equal to 0.51 , directly from the exponential-like maintainability distribution $X$ (those two probabilities have no reason to be the same: the former is related to the strategy -select this particular stoppagewhereas the latter is related to the probability that the stoppage enable to perform the maintenance action). Stoppage 20, which duration is $3.6 \mathrm{~h}$, is preferred to stoppage 19 as the threshold is in this case rather low. With a threshold equal to 0.52 for instance, the twentieth stoppage would be considered a degraded decision by the algorithm, as well as the sixth and the fourteenth stoppages (they all would be eliminated). Stoppage 17 is too short (according to the threshold fixed by the expert) to be considered. The threshold makes choice number 20 (stoppage 2, which should be eliminated) unacceptable as it is. In the same spirit, it can be noticed that the shortest stoppages are penalised in Table 1. This kind of classification of the production stoppages allows the expert to eliminate decisions which could not fit his requirements. For example, with a threshold fixed at 0.53 , the classification would not change, but the decision would be affected: in Table 1, stoppages 20, 16, 14, 12, 7, 6 and 3 would be degraded decisions (symbol * for their probabilities). In that precise case (threshold equal to 0.53 ), the optimal strategy (consider stoppage 20) should be compared with the fact that the corresponding solution 
(stoppage 20) cannot meet the requirements imposed by the expert (the stoppage probability is lower than 0.53). Thus the expert should consider, if the threshold is important for him, the first decision which would not be degraded (stoppage 19, in this particular case). This strategy is not optimal (rigorously speaking) but leads to the stoppage that would best fit the requirements of the expert (select the last production stoppage to perform the maintenance action with expected chances of 0.53 , or, equivalently, with risk lower than 0.47 ). The optimality of the strategy has to be compared to the optimality for the stoppage. The conclusion of this subsection is that a threshold has been introduced in Bruss algorithm to eliminate a priori potential decisions that would be unacceptable for the expert. According to the maintainability alone, the potential stoppages for performing the maintenance action can immediately be found.

\subsection{Results concerning the reliability alone}

The same study as in the previous subsection can be conducted in the case where successes are defined in terms of reliability alone. The characteristics of the stoppages (instants of beginning and durations) are the same as in the previous subsection. The purpose is to choose the last stoppage that will be a success, in terms of reliability, with the threshold imposed by the expert.

\begin{tabular}{|c|c|c|c|c|}
\hline Rank & $\begin{array}{c}\text { Proposed } \\
\text { stoppage }\end{array}$ & $\begin{array}{c}\text { Strategy } \\
\text { probability }\end{array}$ & $\begin{array}{c}\text { Stoppage } \\
\text { probability }\end{array}$ & $\begin{array}{c}\text { Stoppage } \\
\text { beginning } \\
\text { (hours) }\end{array}$ \\
\hline 1 & 19 & $*$ & $*$ & 570 \\
\hline 2 & 18 & $*$ & $*$ & 540 \\
\hline 3 & 17 & $*$ & $*$ & 510 \\
\hline 4 & 16 & $*$ & $*$ & 480 \\
\hline 5 & 15 & 0.51 & 0.52 & 450 \\
\hline 6 & 14 & 0.51 & 0.56 & 420 \\
\hline 7 & 13 & 0.52 & 0.59 & 390 \\
\hline 8 & 12 & 0.53 & 0.63 & 360 \\
\hline 9 & 11 & 0.54 & 0.67 & 330 \\
\hline 10 & 10 & 0.55 & 0.70 & 300 \\
\hline 11 & 9 & 0.56 & 0.74 & 270 \\
\hline 12 & 8 & 0.57 & 0.78 & 240 \\
\hline 13 & 7 & 0.58 & 0.81 & 210 \\
\hline 14 & 6 & 0.59 & 0.85 & 180 \\
\hline 15 & 5 & 0.60 & 0.88 & 150 \\
\hline 16 & 4 & 0.61 & 0.91 & 120 \\
\hline 17 & 3 & 0.62 & 0.94 & 90 \\
\hline 18 & 2 & 0.62 & 0.97 & 60 \\
\hline 19 & 1 & 0.63 & 0.99 & 30 \\
\hline 20 & 20 & $/$ & $/$ & 600 \\
\hline & & & & \\
\hline
\end{tabular}

Table 2. Proposed stoppages for the reliability alone

In Table 2, the threshold has been chosen equal to 0.50 by the expert. This threshold is too high for stoppages 16 to 19 (and moreover for stoppage 20, which should be eliminated): the last stoppages are penalised. Although stoppage 19 is the one that is recommended (rigorously speaking) by the 'odds algorithm', this stoppage, as well as stoppages 16 to 18 , has to be eliminated, should the threshold be important.
Therefore stoppages 1 to 15 can be considered to perform the maintenance action, regarding only the reliability of the component to be maintained. Stoppage 15 is the last one that is a priori convenient for this purpose, with the level of requirements that has been imposed by the expert. Thus the expert should consider, if this level is important, the first decision which would not be degraded (stoppage 15, in this case). Thus the difference between an optimal strategy (consider stoppage 19) and an optimal stoppage for the purpose of the expert (refuse a stoppage whose probability is lower than a fixed threshold) is emphasised. A threshold combined with Bruss algorithm is used to eliminate a priori decisions that would be unacceptable for the expert. According to the reliability alone, the potential stoppages for performing the maintenance action can immediately be found. Any other criterion that would be used to define a success would lead to the same kind of study and conclusion. But another interesting question is the selection of the last production stoppage that would globally be optimal for both maintainability and reliability combined. Such a stoppage would be a non-trivial compromise between two antagonistic criteria (the maintainability function being strictly increasing, and the reliability function being strictly decreasing).

\section{OPTIMALITY WITH RESPECT TO MAINTAINABILITY AND RELIABILITY}

It is proposed in this section to find the last production stoppage that would account for optimality with respect to both reliability of the component at the beginning of the stoppage and maintainability of this component during the stoppage. Indeed the comparison of Tables 2 and 3 leads to the following remarks: stoppages 18 and 19 could be appreciated decisions for both criteria (should the threshold be lower). But stoppage 15, for instance, is optimal for reliability and almost unacceptable as far as maintainability is concerned (whatever the threshold). The proposed decision does not make use of a weighting or a balance between maintainability (cf. 3.2) and reliability (cf. 3.3): they both are considered inside one unique function. Therefore it can be useful to compare the proposed global decisions to those acceptable solutions that have been found with respect to one unique criterion in the previous section. The hypothesis of independence allows the expert to define a success with a threshold applied to a function that is the product of the reliability (of the component at the beginning instant of the stoppage) by the maintainability (of the component during the stoppage). The characteristics used in subsection 3.1 still hold in this section, and the stoppages are the same as previously (see Table 1 for their durations, and Table 2 for their beginning instants).

In Table 3, the threshold has been chosen equal to 0.35 by the expert (which is greater than 0.25 , the product of the previous two thresholds). The adaptation of such a threshold may be hard for the expert in an environment involving a lot of performance criteria. Some further research is needed to help the expert settle this threshold, or to propose a way to eliminate a priori unacceptable solutions that do not make use of such a threshold. With this relatively high level of requirements ( 0.35 for the threshold), the more relevant 
stoppage would be the twelfth. This stoppage was optimal neither with respect to the maintainability, nor with respect to the reliability. However it is optimal for both reliability and maintainability combined. The decisions proposed in this table can be balanced by the expert, by considering the results proposed in the previous two tables: stoppage 10 is supposed to be another relevant solution, but it might be eliminated because of its classification as a degraded decision with respect to the criterion of maintainability alone.

\begin{tabular}{|c|c|c|c|}
\hline Rank & $\begin{array}{c}\text { Proposed } \\
\text { stoppage }\end{array}$ & $\begin{array}{c}\text { Strategy } \\
\text { probability }\end{array}$ & $\begin{array}{c}\text { Stoppage } \\
\text { probability }\end{array}$ \\
\hline 1 & 18 & $*$ & $*$ \\
\hline 2 & 16 & $*$ & $*$ \\
\hline 3 & 15 & $*$ & $*$ \\
\hline 4 & 14 & $*$ & $*$ \\
\hline 5 & 13 & $*$ & $*$ \\
\hline 6 & 12 & 0.42 & 0.35 \\
\hline 7 & 11 & $*$ & $*$ \\
\hline 8 & 10 & 0.42 & 0.35 \\
\hline 9 & 9 & 0.42 & 0.37 \\
\hline 10 & 8 & 0.42 & 0.40 \\
\hline 11 & 7 & 0.43 & 0.44 \\
\hline 12 & 6 & 0.43 & 0.44 \\
\hline 13 & 5 & 0.43 & 0.48 \\
\hline 14 & 4 & 0.43 & 0.46 \\
\hline 15 & 3 & 0.43 & 0.50 \\
\hline 16 & 2 & 0.43 & 0.44 \\
\hline 17 & 1 & 0.43 & 0.53 \\
\hline 18 & 17 & $/$ & $/$ \\
\hline 19 & 19 & $/$ & $/$ \\
\hline 20 & 20 & $/$ & $/$ \\
\hline
\end{tabular}

Table 3. Proposed stoppages for both maintainability and reliability considerations

Stoppages 19 and 20, which were privileged for this same criterion (maintainability), are globally choices to eliminate. Stoppage 15, which was a relevant choice with respect to the reliability, is also a stoppage to eliminate (even with a threshold lower than 0.35 ) because of its duration. However, the expert could decide to wait until stoppage 16 because of extrinsic reasons he would have knowledge of. Stoppage 16 is indeed as long as stoppage 12, although the risk not to be able to carry out the maintenance action is greater with this stoppage (as it is considered a degraded decision both in Tables 2 and 3). At the end of the day, the expert alone will take the decision, aided by the complementary results of Bruss algorithm. The expert can also make use of some of his knowledge by providing convenient thresholds.

\section{CONCLUSION}

Some issues related to the planning of maintenance actions in synchronisation with production have been presented. A maintenance decision support has been proposed and studied in this paper. This tool is proved to be optimal and can be used to propose optimal decisions with respect to some criteria. Maintainability and reliability are the two criteria that have been considered, but any other performance criterion could have been used as well. For each criterion, the optimal decision depends on the expert through the threshold specified by him. Such a threshold enables the integration of the expert's knowledge, the elimination of unacceptable solutions and the proposition of an optimal solution. This answers the question raised in the introduction and is a first step toward the formalisation of the expert's knowledge.

\section{REFERENCES}

Bruss, F.T. (2000). Sum the odds to one and stop. Annals of Probability, 28, pp. 1384-1391.

Bruss, F.T. (2003). A note on bounds for the odds-theorem of optimal stopping. Annals of Probability, 31, pp. 1859-1861.

Chow, Y.S., H. Robbins and D. Siegmund (1991). The theory of optimal stopping. Dover, New York.

Cunha, P.F. and J.A. Caldeira-Duarte (2004). Development of a productive service module based on a life cycle perspective of maintenance issues. Annals of the CIRP, 53 (1), pp. 13-16.

Gharbi, A. and J.-P. Kenné (2005). Maintenance scheduling and production control of multiple-machine manufacturing systems. Computers and Industrial Engineering, 48, pp. 693-707.

ISO 13381-1 (2004). Condition monitoring and diagnostics of machines - Prognostics. ISO.

Iung, B., E. Levrat and E. Thomas (2007). 'Odds algorithm'-based opportunistic maintenance task execution for preserving product conditions. Annals of the CIRP, $\mathbf{5 6}$ (1), to appear.

Iung, B., M. Veron, M.-C. Suhner and A. Muller (2005). Integration of maintenance strategies into prognosis processto decisionmaking aid on system operation. Annals of the CIRP, 54 (1), pp. 5-8.

Kianfar, F. (2005). A numerical method to approximate optimal production and maintenance plan in a flexible manufacturing system. Applied Mathematics and Computation, 170, pp. 924940.

Levrat, E., E. Thomas and B. Iung (2007a). 'Odds'-based decisionmaking tool for opportunistic production-maintenance synchronisation to preserve component performances. Submitted to: International Journal of Production Research.

Levrat, E., E. Thomas and B. Iung (2007b). A decision tool for opportunistic maintenance strategy to preserve component performances. In: IFAC conference on Cost Effective Automation (CEA) in networked product development and manufacturing, $2^{\text {nd }}-5^{\text {th }}$ October 2007, Monterrey, Mexico (Accepted).

Rosqvist, T. (2002). Stopping time optimisation in condition monitoring. Reliability Engineering and System Safety, 76, pp. 319-325.

Takata, S., F. Kimura, F.J.A.M. Van Houten, E. Westkämper, M. Shpitalni, D. Ceglarek and J. Lee (2004). Maintenance: changing role in life cycle management. Annals of the CIRP, $\mathbf{5 3}$ (2), pp. 643-656.

Thomas, E., E. Levrat, B. Iung and M. Monnin (2006). 'Odds Algorithm'-based opportunity-triggered preventive maintenance with production policy. In: Proceedings of the 6th IFAC Symposium on Fault Detection, Supervision and Safety of Technical Processes, SAFEPROCESS'06, pp. $379-384,29^{\text {th }}$ August $-1^{\text {st }}$ September 2006, Beijing, P. R. China.

Thomas, E., E. Levrat and B. Iung (2007). Bruss algorithm as a contribution to opportunistic preventive maintenance. In: $2^{\text {emes }}$ Journées Doctorales / Journées Nationales 'Modélisation, Analyse et Conduits des Systèmes dynamiques' JD-JN MACS, $9^{\text {th }}-11^{\text {th }}$ July 2007, Reims, France (In French).

Van Houten, F.J.A.M., T. Tomiyama and O.W. Salomons (1998). Product modelling for model-based maintenance. Annals of the CIRP, 47 (1), pp. 123-129. 